\title{
Emerging Role of Aurora A in Radioresistance: A Comprehensive Review
}

\author{
Authors: \\ Salini Das, Elizabeth Mahapatra, Souvick Biswas, Madhumita Roy, \\ *Sutapa Mukherjee \\ Department of Environmental Carcinogenesis and Toxicology, Chittaranjan National \\ Cancer Institute, Kolkata, India \\ *Correspondence to sutapa_c_in@yahoo.com \\ Disclosure: $\quad$ The authors have declared no conflicts of interest. \\ Received: $\quad 17.10 .20$ \\ Accepted: $\quad 10.05 .21$ \\ Keywords: Aurora A, cancer, radioresistance. \\ Citation: $\quad$ EMJ Oncol. 2021;9[1]:81-90.
}

\begin{abstract}
Radiotherapy is one of the most conventional modes of treatment in several cancers. Failure of radiotherapy followed by acquisition of radioresistance is one of the emerging challenges faced by clinical experts. Unusual expression and functional implications of several molecules are observed to facilitate radioresistance. Aurora A, a member of the Aurora kinase (serine/threonine kinase) family, is one such molecule that shows significantly altered expression as well as non-canonical functional crosstalk with other associated factors (cell cycle regulators, signaling molecules, stemness markers, etc.) to favour the adaptations for the acquirement of radioresistance. These mechanisms include progression of cell cycle, stimulatory activation of factors by phosphorylation for enhancing the chance of cellular survivability, and prevention of apoptosis. This review article summarises how Aurora $A$ is responsible for radioresistance in cancer and why this kinase should be considered a negative biomarker of radiosensitivity. This review discloses a wider opportunity in the field of research to find the mechanistic key regulatory pathway of Aurora A, which can be a potential target for enhancing the efficiency of treatment. Further investigations are required to explore the potential of Aurora $A$ inhibitors as reliable radiosensitisers.
\end{abstract}

\section{INTRODUCTION}

Radioresistance is known to create complications in the treatment of cancer. ${ }^{1}$ Radiation-induced altered adaptive responses by tumour cells or tissues are considered to be primary reasons behind the failure of radiotherapy. ${ }^{2}$ Acquirement of radioresistance followed by treatment failure and locoregional recurrence is a multifaceted process. Emerging data have suggested various molecular biomarkers necessitate radioresistance in cancer. ${ }^{3,4}$ It is well established that the level of

radioresistance alters in different phases of the cell cycle. Several researchers have experimentally proven that most cells are resistant in the GO phase, early $\mathrm{G} 1$ phase, and late $\mathrm{S}$ phase of the cell cycle. In contrast, most cells are radiosensitive in the late $\mathrm{G} 1$ phase, $\mathrm{G} 2$ phase, and throughout the $M$ phase of the cell cycle. ${ }^{5}$ In the $S$ phase, radiation resistance is thought to be due to an increased amount of DNA synthesis and repair enzymes, as well as a rise in the intracellular levels of glutathione (a free radical scavenger). In response to ionising radiation, the $\mathrm{G} 1$ phase of 
the cell cycle is generally blocked to allow time for the recognition and repair of DNA damage prior to the initiation of DNA synthesis. Cells show the most sensitivity towards radiation during the G2/M phase of the cell cycle because of the lack of time for adequate repair before chromosome segregation. The key participating genes and their products halting cell cycle progression, which increase or are post-translationally altered following DNA damage, are p53, p21, growth arrest and DNA damage-inducible protein 45 , and the retinoblastoma protein. Overall, there is a damageresponsive $\mathrm{G} 1$ block, halting the cell cycle at $\mathrm{G} 2 / \mathrm{M}$ until DNA damage can be repaired. Thus, those cells having the ability to repair the damaged DNA may improve cell survival and impart additional resistance towards radiation. ${ }^{6,7}$ It is widely reported that the essential mechanism by which ionising radiation exerts its therapeutic effect is by induction of DNA damages, such as doublestrand breaks, single-strand breaks, and oxidation of DNA bases. These damages may be removed by homologous recombination, non-homologous end joining, single-strand break repair, and base excision repair. ${ }^{8}$ Now, it is of importance to note why and how these participating genes get altered following DNA damage. Aurora $A$ is found to interact with most of these regulators of cell cycle checkpoints to override their effect and continue cell cycle progression, and thereby contributes to radioresistance.
Three families of human Aurora kinases, Aurora kinase $A, B$, and $C$, share a common C-terminal catalytic domain in their proteins. ${ }^{9}$ These three kinases are known to participate in mitotic progression of cells; however, little is known about Aurora kinase C. ${ }^{9}$ During mitosis, centrosome maturation and separation followed by the assembly and stability of spindle is controlled by Aurora Kinase $A .{ }^{10}$ Aurora kinase $B$ is a member of the chromosomal passenger complex along with other members (survivin, borealin, and INCENP)." This kinase allows proper segregation of chromosomes and completes the cytokinesis process." Evidence suggests that the zone of Aurora kinase B localisation is at the K-fibres, which are the specialised microtubules near kinetochores;1 however, this localisation varies in different phases of the cell cycle, and is at the chromosomes in prophase, the centromere in prometaphase and metaphase, and the central mitotic spindle in anaphase..$^{12}$ Although Aurora kinase A and B are found in most of the somatic cells, Aurora kinase $C$ is distributed in a limited manner to germline cells (sperm and oocyte) undergoing meiosis. ${ }^{13}$ Aurora $\mathrm{C}$ primarily acts as a regulator of chromosome segregation in a lesser-known mechanism. The cell cycle specific distributions of these kinases are summarised in Figure 1.

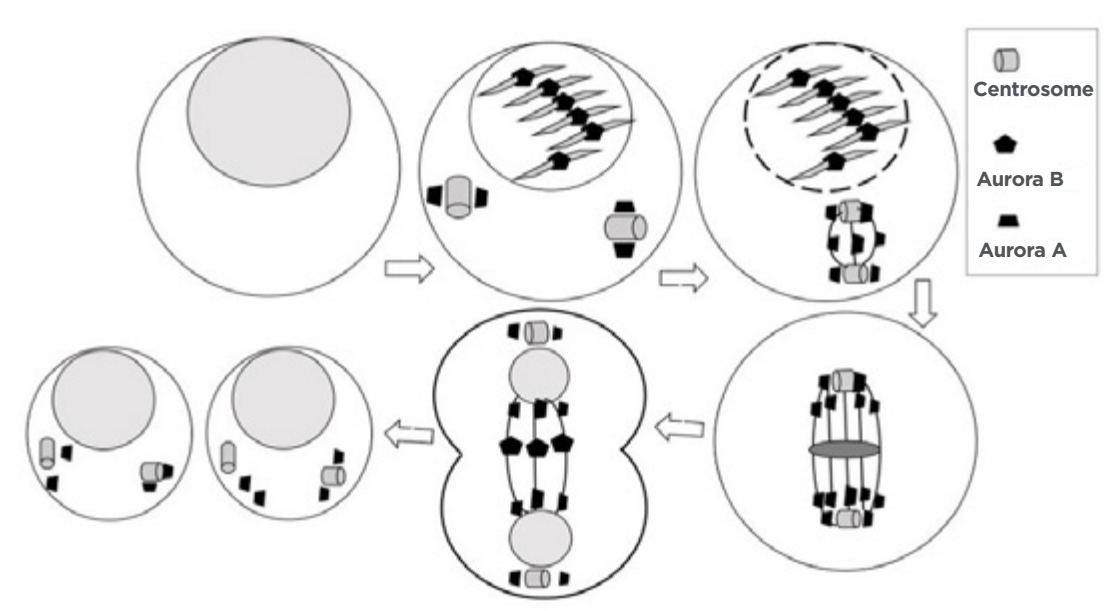

Figure 1: Cell cycle phase-specific distribution of Aurora kinases.

Aurora A localises to the centrosome from G1/S phase, with the progression of cell cycle it localises in spindle poles until telophase. Aurora B localises to the kinetochore followed by midbody of the central spindle during mitosis. The progression of cell division is shown using arrow signs from left showing normal cell, cover by onell in early prophase, cell in late prophase, cell in metaphase, cell in anaphase, and cells after division. 
Apart from the canonical activities regulated by Aurora A, its overexpression also correlates with acquired radioresistance in several cancers. This review highlights some of the mechanisms performed by Aurora A in imparting radioresistance in cancer.

\section{AURORA A}

\section{Salient Structural Features}

Aurora A (or STK15/BTAK) is a well-known cell cycle regulatory kinase. Being a mitotic kinase, it is a member of the serine/threonine protein kinase family, which plays an important role in proper entry of cells in mitosis, formation of a bipolar spindle, control of centrosome maturation, and appropriate chromosomal segregation during mitosis. ${ }^{8}$ If the activity of Aurora $A$ is suppressed by RNA interference, it results in delayed mitotic entry of cells, ${ }^{14}$ whereas centrosome amplification, cytokinesis inhibition, and aneuploidy are some well-verified aftereffects of its overexpression. ${ }^{15}$ Before looking into the functional mechanism of Aurora $A$, it is essential to understand the different protein domains of this kinase. The Aurora A gene has its chromosomal location at 20q13.2, which is often found to be significantly amplified in several human epithelial tumours. ${ }^{16}$ The molecular weight of the mammalian Aurora A protein is $46 \mathrm{kDa}$ and the protein is 402-403 amino acids long. The common structural configuration of all Aurora kinases includes an $\mathrm{N}$-terminal domain (39-139 amino acids), a kinase domain (250-300 amino acids), and a C-terminal domain (15-20 amino acids). ${ }^{17}$ The kinase domain of Aurora $A$ is mainly composed of a $\beta$-stranded $N$-terminal lobe and $\alpha$-helical $C$-terminal lobe that are linked together by a hinge region in order to acquire the active conformation. ${ }^{18}$ The ATP-binding domain of Aurora A consists of three specific sequence variants (leucine 215, threonine 217, and arginine 220), of which the threonine 217 locus particularly distinguishes Aurora A from Aurora B kinase domains. ${ }^{19}$ These particularities of the Aurora A domain are useful for the design of specific Aurora A inhibitors. Some non-catalytic domains present in Aurora A trigger its degradation. These include the D-boxes, KEN motifs, and the DAD/A boxes. Mutation of the C-terminal D-box sequence is essential for the stability of Aurora A, and degradation of Aurora A depends on D-Box instead of KEN-box motifs (residues 6-9). Aurora A degradation is also mediated by the anaphase promoting complex or cyclosome, which occurs at an atypical degradation sequence present in Aurora $A$ named the DAD/A box. ${ }^{20,21}$ Aurora A possesses a short $\alpha$-helix called the B-helix, located just prior and perpendicular to the C-helix. The structure of Aurora A is depicted in Figure 2.

The C-terminal region of the kinase domain consists of seven $\alpha$-helices and a two-stranded $\beta$-sheet, and contains the catalytic aspartic acid of the HRD motif (sequence histidine, arginine, and aspartate at positions 254-256) and the mobile activation loop, whose position and conformation determine whether a kinase is active or inactive. ${ }^{22,23}$ The Aurora A activation loop (the most crucial part required for functional activation of the protein) spans 274-299 amino acid residues, beginning with an aspartate, phenylalanine, and glycine sequence motif and ending with an alanine, proline, and glutamate motif (sequence proline, proline, and glutamate in Aurora A). Aurora A becomes active by its autophosphorylation (discussed in detail later) and trans-phosphorylation at threonine 287 and threonine 288 residues by targeting protein for Xenopus kinesin-like protein 2,24 p21 activated kinase, ${ }^{25}$ protein kinase $A,{ }^{26}$ or atypical protein kinase $C^{27}$

\section{Localisation}

Being a centrosomal protein, Aurora A resides next to the centrosome late in the $\mathrm{G} 1$ phase and early in the $\mathrm{S}$ phase. The $\mathrm{N}$-terminal domain of Aurora A participates in the localisation of the kinase to the centrosome during interphase. With the progression of the cell cycle, Aurora A concentrations increase and an association of the kinase with the mitotic poles and the adjacent spindle microtubules is observed. Such association lasts until telophase. Re-localisation of Aurora $A$ to the mid-zone of the spindle occurs immediately before mitotic exit. ${ }^{28}$

\section{AURORA A ACTIVATION}

\section{Transcriptional}

The cell cycle-induced transcription of Aurora proteins is made possible by the presence of the cell cycle-dependent element and cell cycle gene homology region in Aurora A promoters. 


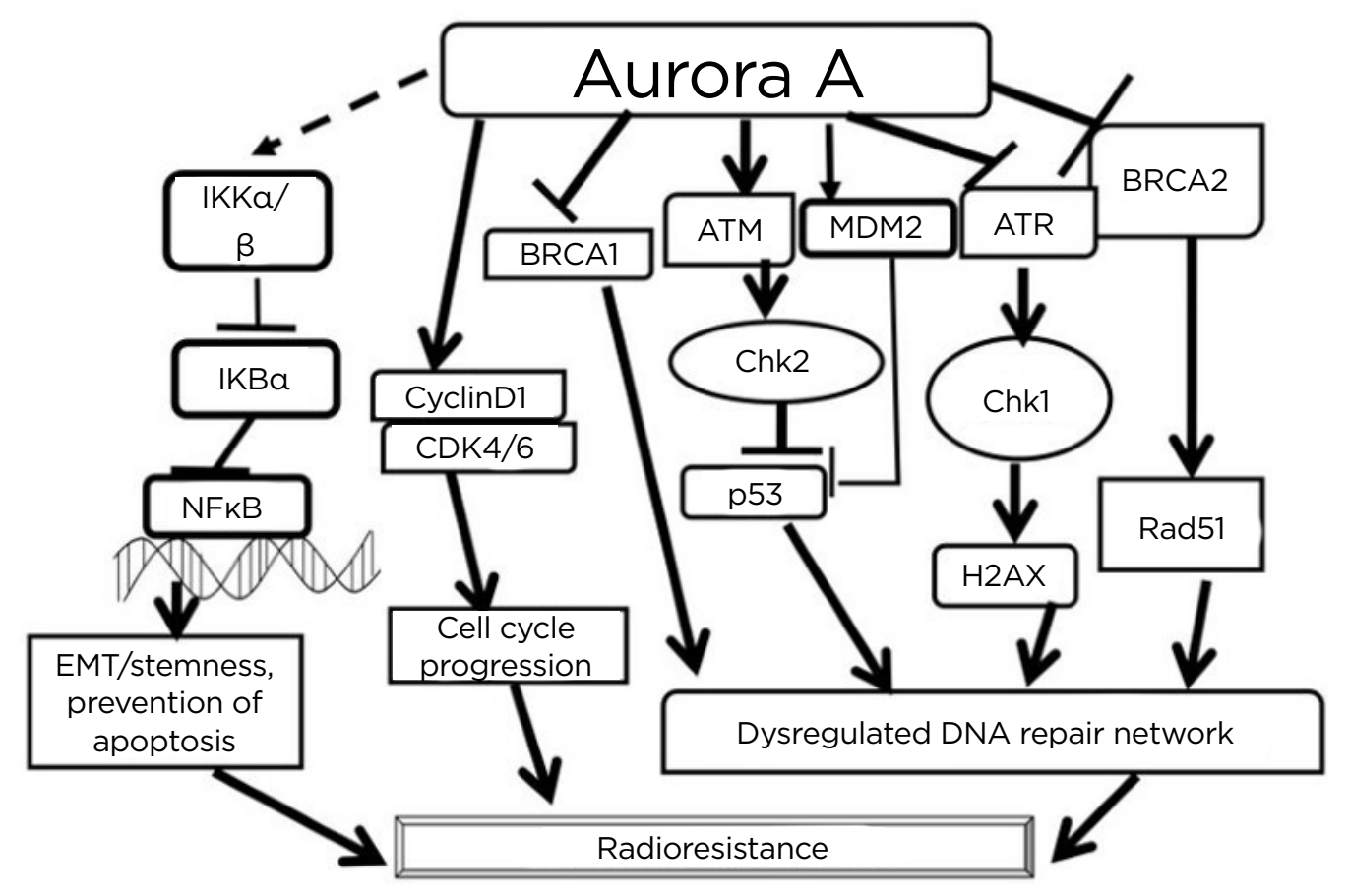

Figure 2: Some of the well-studied interactions of Aurora $A$ that are found to help in acquirement of radioresistance. Aurora A activates NFKB and generates EMT and causes prevention of apoptosis. The kinase can activate cyclin D1 and CDK to allow progression of cell cycle. Aurora A, by interacting with DNA repair regulators (BRCA1, BRCA2, ATM and Rad3-related kinase, Chk 1/2, and p53), causes dysregulated DNA repair. All of these result in changes that lead to radioresistance.

ATM: ataxia-telangiectasia-mutated kinase; ATR: ataxia-telangiectasia-mutated and Rad3-related kinase; BRCA: breast cancer susceptibility protein; CDK4/6: cyclin-dependent kinase 4/6; Chk: checkpoint kinase; EMT: epithelialmesenchymal transition; MDM2: mouse double minute 2 homolog.

The cell cycle-dependent element and cell cycle gene homology region sequences in Aurora $A$ mediate the transcription of Aurora $A$ and other crucial G2/M regulators (e.g., cyclin A, cell division cycle 25 phosphatase, cyclin-dependent kinase $[C D K] 1$, and polo-like kinase). ${ }^{29}$

\section{Post-translational Modifications}

Post-translational modification is an important prerequisite for functional activation of the enzyme. Like other serine/threonine kinases, functional regulation of Aurora A occurs through activation loop phosphorylation. Aurora A autophosphorylation, a salient post-translational modification, is mediated by several co-factors, of which Ajuba, targeting protein for Xenopus kinesin-like protein 2, protein aurora borealis, and transforming acidic coiled-coil-containing protein 3 are the noteworthy ones. As described earlier, Aurora A has two regulatory sites for phosphorylation in its activation loop (threonine 287 and threonine 288). Phosphorylation of Aurora $A$ in its catalytic domain at threonine 288 is known to trigger the kinase activity of the enzyme. ${ }^{24}$ This phosphorylation eventually causes a positive feedback loop, which is responsible for maintaining the activated state of the kinase until anaphase. Maximum activity has been observed from late $\mathrm{G} 2$ until pro-metaphase. The function of threonine 287 phosphorylation is still unclear. ${ }^{30}$

\section{RADIORESISTANCE IN CANCER: FUNCTIONAL ROLE OF AURORA A}

Apart from its intricate role in regulating mitosis, activated Aurora A also subsequently promotes 
a variety of biological functions for maintaining cancer phenotypes. Some of the functions of Aurora A include cell proliferation, migration, invasion, epithelial-mesenchymal transition, and maintenance of cancer stem cell behaviors. ${ }^{31}$ Now, it is necessary to understand whether Aurora A has any impact on radiotherapy in cancer. Ectopic expression of Aurora A led to increased sensitivity to ionising radiation in MCF10A normal breast epithelial cells. ${ }^{30}$ In contrast, in cancer cells, a plethora of evidence suggests that excessive Aurora A attenuates the radiosensitivity of cells, while silencing or inhibition of Aurora A with small interfering RNA or selective inhibitors enhances radiosensitivity. ${ }^{32}$ The possibility of involvement of Aurora $A$ in contributing radioresistance has been experimentally tested in several cancers. Overexpression of Aurora A in cervical squamous cell carcinoma showed a strong positive correlation with cancer cell invasion and metastasis. Furthermore, Aurora $A$ is considered to serve as an independent prognostic factor, which affects disease-free survival and overall survival. ${ }^{33}$ In one study, patients with cervical cancer were treated with Aurora A inhibitors. Treatment outcomes included increased cell apoptosis after $\mathrm{X}$-irradiation and downregulation of the expression of cyclin D1, CDK2, and CDK6, which eventually induced cell cycle arrest at the $G 2 / M$ phases. ${ }^{34}$ These findings indicate that Aurora A activation gives rise to radiation resistance by allowing cell cycle progression in cervical cancer. Therefore, Aurora A may be regarded as one of the therapeutic targets and an independent prognostic factor for increasing the sensitivity of cervical squamous cell carcinoma radiotherapy. A predominant finding of Shen et al., who studied hepatocellular carcinoma (HCC) cell lines, revealed lower expression of nuclear $\mid \kappa \mathrm{B} \alpha$ protein in parental HCC cells at the same time as higher expression levels of p65 protein in radioresistant counterparts. Knockdown of Aurora A led to increased expression of nuclear $I_{\kappa} \mathrm{B} \alpha$ proteins by way of decreased expression of p65 proteins in radioresistant HCC cells. The expression of downstream effectors of the NF$\kappa B$ pathway, such as B-cell lymphoma 2 , myeloid cell leukaemia 1, cleaved poly(ADP-ribose) polymerase, and caspase-3 (cysteine-aspartic proteases), is enhanced by hyperproduction of Aurora A. Triggering apoptosis in radioresistant cells was attained by knockdown of Aurora $A$, which resulted in downregulated B-cell lymphoma 2 and myeloid leukaemia 1 protein expressions as well as upregulated cleaved poly(ADP-ribose) polymerase and caspase-3 expressions in radioresistance. ${ }^{35}$ To explore whether Aurora A contributes to radioresistance, a study was conducted by Sun et al., using Aurora A complementary DNA/short hairpin RNA or the specific inhibitor VX-680 (Vertex Pharmaceuticals, Inc., Boston Massachusetts, USA). This study confirmed that Aurora A positively regulates cell proliferation, cell cycle progression, and anchorage-independent cell growth in order to establish resistance against $X$-rays. Simultaneous promotion of the expression of ataxia-telangiectasia-mutated kinase/checkpoint kinase 2 and suppression of the expression of breast cancer susceptibility protein 1/2, ataxia-telangiectasia-mutated and Rad3-related kinase/checkpoint kinase 1, p53, phospho-p53 (serine 15), H2AX, $\gamma \mathrm{H} 2 \mathrm{AX}$ (serine 139), and RAD51 by Aurora A resulted in a dysregulated DNA repair mechanism. The formation of a $\gamma \mathrm{H} 2 \mathrm{AX}$ focus, which is considered as one of the prognostic markers of radiationinduced DNA damage, was found to be minimised by Aurora $A$. These reports account for supportive evidence of the hypothesis that Aurora $A$ is a negative biomarker of radiosensitivity. ${ }^{36}$ Similar results were observed in HeLa cells, which were irradiated with $4 \mathrm{~Gy}$ of $\gamma$-rays. The electrophoretic mobility shift assay, luciferase reporter gene assay, immunoblot analysis, small interfering RNA-based gene knockdown, and overexpression studies concluded that Aurora A enhances the binding of $N F-\kappa B$ to DNA, thereby increasing the gene transcription by NF- $\mathrm{KB}$ and decreasing the radiosensitivity of the cells. ${ }^{37}$ Equivalent results were obtained in radioresistance of lung adenocarcinoma. ${ }^{38}$ The effectiveness of radiationinduced cell death often relies on the mechanism of reactive oxygen species-mediated cellular damage. Tumour tissues that are deprived of adequate oxygen supply due to hypoxic conditions lack sufficient amounts of reactive oxygen species, thereby causing a three-fold increase in the chance of acquired radioresistance. The transformed tumor cells get adapted in the hypoxic tumour microenvironment by developing resistant characters in them. ${ }^{39}$ Simultaneous overexpression of hypoxia-inducible factor$1 \alpha$ and Aurora $A$ is a remarkable event during hypoxia, which establishes a positive feedback between these two molecules. ${ }^{40}$ It has been 
suggested that hypoxic cellular proliferation and migration are clinically correlated with increased mRNA and protein expression of Aurora A. Conversely, inhibition of Aurora A could reverse this event. ${ }^{40}$ In a further study, it was confirmed that in hypoxia, hypoxia-inducible factor-1 $\alpha$ binds at the promoter region of Aurora $A$ and enhances its transcription. ${ }^{41}$ This associative involvement of Aurora $A$ in both hypoxia and radioresistance is notable, which again provokes the possibility of this kinase in imparting radioadaptive responses during acquirement of radioresistance. Experimental evidence indicated that MLN8237 (Takeda Pharmaceutical Company Ltd., Tokyo, Japan), a selective Aurora A inhibitor, could induce cell cycle arrest at the $G 2 / M$ phase and significantly reduce radiation-dependent resistance. ${ }^{42}$ Similar studies were found in selected lung cancer cell lines, where inhibition of Aurora A enhanced radiosensitivity. ${ }^{43}$ Increased expression of Aurora $A$ is negatively correlated with survival in patients with non-small cell lung carcinoma. ${ }^{43}$ One of the established Aurora kinase inhibitors, daurinol, targets the kinase and enhances radiotherapy; ${ }^{44}$ however, the exact mechanism concerning the functional impact of this Aurora A inhibitor needs to be investigated thoroughly. Whether the inhibitor enhances radiosensitivity by directly blocking Aurora A or through inhibiting cyclin expressions (cyclin D1, CDK2, and CDK6) is yet to be elucidated. ${ }^{44}$ Aurora kinase expression was reported to be regulated transcriptionally by radiation because the mRNA and protein expression of such kinases were increased by sub-lethal doses of radiation. ${ }^{45}$ The level of Aurora kinases present in pre-treatment biopsies could serve as a predictive factor to identify patients likely to respond to conservative radiotherapies. Pharmacological approaches targeting Aurora kinases in tumors over-expressing these proteins could strongly increase the therapeutic ratio in radiotherapy for cancer treatment. The underlying mechanisms by which targeting aurora kinases may improve the response to radiation seem to be multifaceted and involves cell cycle distribution. ${ }^{43}$ In radioresistant pancreatic cancer cells, treatment with an Aurora A inhibitor resulted in co-inhibition of cyclin D1, CDK2, and CDK6 to induce cell cycle arrest at the $\mathrm{G} 1 / \mathrm{S}$ and $\mathrm{G} 2 / \mathrm{M}$ phases, and also promoted cell apoptosis after $\gamma$-irradiation. ${ }^{46}$ In laryngeal cancer cells, inhibiting Aurora $A$ by $V X-680$ induced expression of p53 and potently sensitised cells to radiotherapy, leading to significant cell death, whereas ectopic overexpression of Aurora A reduced p53 levels and rendered cells more resistant to irradiation. Taken together, Aurora A kinase, a negative prognostic marker, promotes migration, and reduces radiosensitivity. ${ }^{47}$ The Aurora A signaling axis pertinent to radioresistance is represented in Figure 2.

\section{OTHER PROBABLE INTERACTIONS MEDIATED BY AURORA A IN RADIORESISTANCE}

Radioresistance is a collective contribution of several factors, with which Aurora A interacts. Signaling pathways like phosphatidylinositol 3-kinase/protein kinase $\mathrm{B}, \mathrm{NF}-\mathrm{kB}$, and Wnt/ $\beta$-catenin in association with stemness markers like sex-determining region Y-box 2, octamer-binding transcription factor 4 , and Twist1 guide the cells to obtain epithelialmesenchymal transition-like characteristics, which accounts for stabilisation of the resistant phenotype. ${ }^{48-59}$ Aurora $A$ is found to interact with these molecules during cancer progression. Therefore, there are strong possibilities that such interactions play an essential role in Aurora A-mediated radioresistance. Some of these interactions are summarised in Table 1.

\section{CONCLUSIONS AND PERSPECTIVES}

One of the major reasons behind the therapeutic failure of radiation is radioresistance. Cancer cells that survive the effects of radiation acquire adaptations to bypass cell cycle checkpoints and thereby retain their proliferative and invasive properties. Several faulty repair mechanisms accumulate changes in sequences of DNA, which makes this process smoother. The regulatory role of Aurora $A$ in mitotic and non-mitotic events involves several molecules, ranging from DNA repair mediators to signaling modulators. It is more important to highlight that blocking and inactivation of Aurora A activity can reverse the process of 
radioresistance and increase radiosensitivity. Treatment of patients using fractionated irradiation determines acquirement of resistance against radiotherapy, which is clinically complemented with overexpression of Aurora A. Thereby, Aurora A can serve as a prognostic marker of radioresistance. This review provides a thought-provoking element to global researchers, encouraging them to design novel experiments in order to explore the mechanistic key regulatory pathway of Aurora A necessary for enhancing the efficiency of treatment. Checking the levels of Aurora A along with administration of Aurora A inhibitors prior to radiotherapy could be applied in the future to enhance the efficacy of radiotherapy. Clinical administration of Aurora A inhibitors as reliable radiosensitisers may serve as a scope of future investigations. The details of several Aurora kinase inhibitors are provided below.

\section{Type of Inhibitor}

\section{Pan-aurora inhibitor}

Tozasertib (VX-680/MK0457 [Merck \& Co., Kenilworth, New Jersey, USA]) is currently in Phase II clinical trials, and exerts its activity by inducing apoptosis and autophagy. ${ }^{60}$ Danusertib (PHA-739358), which is also in Phase II clinical trials, blocks the activities of fibroblast growth factor receptor 1, Abl, rearranged during transfection, and tropomyosin receptor kinase A, and increases expression of the p53 protein and its downstream effector protein p21. ${ }^{61}$

Table 1: Interactions of several factors with Aurora A that have a potential to generate radioresistance.

\begin{tabular}{|c|c|c|c|}
\hline Name of the factor & Interaction with Aurora A & Result & Reference(s) \\
\hline PI3K/Akt & $\begin{array}{l}\text { Aurora A phosphorylates Akt at its } \\
\text { activation site (serine } 473 \text { ). }\end{array}$ & $\begin{array}{l}\text { Akt activation eventually activates } \\
\text { mTOR pathway, thereby enhancing } \\
\text { radioresistance by regulating the level } \\
\text { of EMT-related markers. }\end{array}$ & 42 \\
\hline sox2 & $\begin{array}{l}\text { Aurora A phosphorylates SOX } 2 \text { at } \\
\text { two important phosphorylation sites: } \\
\text { serine } 220 \text { and serine } 251 \text {. }\end{array}$ & $\begin{array}{l}\text { Maintenance of cancer stem cell } \\
\text { properties and reduced sensitivity } \\
\text { towards radiation. }\end{array}$ & 43 \\
\hline Twist1 & $\begin{array}{l}\text { Aurora A phosphorylates Twist1 at } \\
\text { three sites (serine 123, threonine 148, } \\
\text { and serine 184) and stabilises it upon } \\
\text { binding. } \\
\text { A reciprocal interaction by Twist1 is } \\
\text { observed because it prevents Aurora A } \\
\text { degradation. }\end{array}$ & $\begin{array}{l}\text { Both the proteins take part in EMT } \\
\text { progression. Expression of Twist } 1 \text { is } \\
\text { usually high in radioresistant cancer } \\
\text { cells. }\end{array}$ & $44-45$ \\
\hline Wnt/B-catenin pathway & 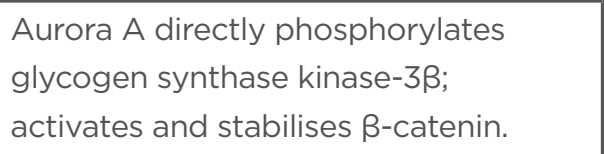 & $\begin{array}{l}\text { The Wnt/B-catenin pathway is a major } \\
\text { pathway linked with the formation of } \\
\text { cancer radioresistance. }\end{array}$ & 46 \\
\hline Myc & $\begin{array}{l}\text { Aurora A activates c-Myc transcription } \\
\text { by interaction with the CCCTCCCCA } \\
\text { motif in the NHE III region. c-Myc } \\
\text { also acts as transcriptional activator } \\
\text { of Aurora A via physical binding to } \\
\text { Aurora A promoter in a non-canonical } \\
\text { E box, which represents potential } \\
\text { Myc binding site ( } 668 / 400 \text { region of } \\
\text { Aurora A promoter). }\end{array}$ & $\begin{array}{l}\text { Dysregulation of the DNA repair } \\
\text { pathway. Myc also maintains GSH and } \\
\text { ROS levels to increase the cancer stem } \\
\text { cell-like population and properties, } \\
\text { therefore conferring radioresistance. }\end{array}$ & 47 \\
\hline
\end{tabular}




\begin{tabular}{|l|l|l|l|}
\hline $\begin{array}{l}\text { Long, non-coding RNA } \\
\text { (MALAT1, TUG1) }\end{array}$ & $\begin{array}{l}\text { Long, non-coding RNAsare prospective } \\
\text { transcriptional regulators, which } \\
\text { work in association with chromatin } \\
\text { remodeling complex to regulate the } \\
\text { expression of Aurora A. }\end{array}$ & $\begin{array}{l}\text { Long, non-coding RNA knockdown } \\
\text { enhances radiosensitivity of cancer. }\end{array}$ \\
\hline $\begin{array}{l}\text { MicroRNA (microRNA- } \\
\text { 4715-3p; microRNA-129- } \\
\text { 3p; microRNA-34/449) }\end{array}$ & $\begin{array}{l}\text { MicroRNAs block the transcription of } \\
\text { of Aurora A by interacting at the 3'UTR } \\
\text { expression. }\end{array}$ & $\begin{array}{l}\text { Functional inactivation of microRNAs } \\
\text { cause bypass of cell cycle and initiates } \\
\text { radioresistance. }\end{array}$ & 52-53 \\
\hline
\end{tabular}

Akt: protein kinase B; EMT: epithelial-mesenchymal transition; GSH: glutathione; MALAT7: metastasis-associated lung adenocarcinoma transcript 1; mTOR: mechanistic target of rapamycin; NHE III1: nuclease hypersensitive element III1 region; PI3K: phosphatidylinositol 3-kinase; ROS: reactive oxygen species; SOX2: sex determining region Y-box 2; TUG1: taurine up-regulated 1; 3'UTR: three prime untranslated region.

PHA-680632, CYC-116, SNS-314, R763, and AMG-900 are currently in Phase I clinical trials. PHA-680632 shows additive effects in cancer cells in association with radiation. Treatment with PHA-680632 prior to ionising radiation causes enhancement in apoptosis, micronuclei formation, and breast cancer susceptibility protein 1 foci formation. ${ }^{62}$ CYC116 inhibits Aurora activity by interfering with its autophosphorylation, reducing histone $\mathrm{H} 3$ phosphorylation and subsequent polyploidy, and ultimately causing failure in cytokinesis followed by cell death. ${ }^{63}$ SNS-314 exhibits potent and sustained responses, including reduced phosphorylated histone $\mathrm{H} 3$ levels, increased caspase-3, and appearance of increased nuclear size. ${ }^{64}$ R763 causes enlargement of cell size, endoreduplication, and apoptosis. ${ }^{65}$ AMG-900 is an ATP-competitive phthalazinamine small molecule inhibitor of Aurora kinases. AMG 900 inhibits autophosphorylation of Aurora kinase and phosphorylation of histone $\mathrm{H} 3$ on serine 10. This leads to aborted cell division without a prolonged mitotic arrest, which ultimately results in cell death. ${ }^{66}$ AT-9283 is currently in Phase II clinical trials, and promotes a clear polyploid phenotype by inhibiting the activity of Aurora kinase. ${ }^{67}$ Finally, PF-03814375, which is in Phase I clinical trials, is a novel, potent, orally bioavailable, reversible inhibitor of Aurora kinase. ${ }^{68}$

\section{Aurora A inhibitor}

Alisertib (MLN8237), which is in Phase II clinical trials, performs cell cycle arrest at the $G 2 / M$ phase, and instigates apoptosis and senescence. It allows up-regulation of p53, p21, and p27, and cleavage of poly(ADP-ribose) polymerase, caspase-3, and caspase-9. ${ }^{69}$ ENMD-2076 is also in Phase II clinical trials, and inhibits the activity of Aurora A and B kinases, as well as inducing

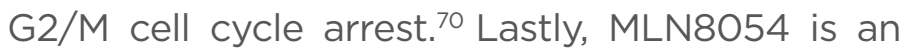
ATP-competitive, reversible inhibitor of Aurora A kinase. Its mechanism of actions includes halting cell proliferation by promoting $G 2 / M$ accumulation and spindle defects. A recent study showed that MLN8054 sensitises androgenresistant prostate cancer to radiation by inhibiting Aurora A kinase, which is associated with sustained DNA double-strand breaks. ${ }^{71,72}$

\section{References}

1. Murata $\mathrm{K}$ et al. Understanding the mechanism underlying the acquisition of radioresistance in human prostate cancer cells. Oncol Lett. 2019;17:5830-8.

2. Norman CC et al. Radiation-induced adaptive response: new potential for cancer treatment. Clin Cancer Res. 2020;26(22):5781-90.
3. Kilic $\mathrm{S}$ et al. The relevance of molecular biomarkers in cervical cancer patients treated with radiotherapy. Ann Transl Med. 2015;3(18):261.

4. Akervall $\mathrm{J}$ et al. A novel panel of biomarkers predicts radioresistance in patients with squamous cell carcinoma of the head and neck. Eur J Cancer. 2014;50(3):570-81.
5. Mcllwrath AJ et al. Cell cycle arrests and radiosensitivity of human tumor cell lines: dependence on wild-type p53 for radiosensitivity. Cancer Res. 1994;54(14):3718-22

6. Otani $\mathrm{K}$ et al. Cell-cycle-controlled radiation therapy was effective for treating a murine malignant melanoma cell line in vitro and in vivo. Sci Rep. 2016;6:30689. 
7. Sharda N et al, "Radiation resistance," Bertino J (ed.), Encyclopaedia of Cancer (2002) 2nd edition: Academic Press, pp.1-11.

8. Biau $\mathrm{J}$ et al. Altering DNA repair to improve radiation therapy: specific and multiple pathway targeting. Front Oncol. 2019;9:1009.

9. Goldenson B et al. The Aurora kinases in cell cycle and leukemia. Oncogene. 2015;34:537-45.

10. Barr RA et al. Aurora-A: the maker and breaker of spindle poles. J Cell Sci. 2007;120:2987-96.

11. Krenn et al. The Aurora B kinase in chromosome bi-orientation and spindle checkpoint signaling. Front Oncol. 2015;5:225

12. Adams RR et al. Chromosomal passengers and the (Aurora) ABCs of mitosis. Trends Cell Biol. 2001;11(2):49-54

13. Quartuccio SM et al. Functions of Aurora kinase $C$ in meiosis and cancer. Front Cell Dev Biol. 2015;3:50.

14. Magnaghi-Jaulin L et al. Aurora A protein kinase: to the centrosome and beyond. Biomolecules. 2019;9(1):28.

15. Asteriti IA et al. The Aurora-A inhibitor MLN8237 affects multiple mitotic processes and induces dosedependent mitotic abnormalities and aneuploidy aneuploidy. Oncotarget. 2014:5(15):6229-42

16. Wang $X$ et al. Overexpression of Aurora-A kinase promotes tumor cell proliferation and inhibits apoptosis in esophageal squamous cell carcinoma cell line. Cell Res. 2006;16:356-66.

17. Lin YS et al. Gene expression profiles of the aurora family kinases. Gene Expr. 2006;13(1):15-26.

18. Siddhanta $\mathrm{S}$ et al. Surface enhanced Raman spectroscopy of Aurora kinases: direct, ultrasensitive detection of autophosphorylation. RSC Adv. 2013;3:4221-30.

19. Cheetham GMT et al. Crystal structure of Aurora-2, an oncogenic serine/threonine kinase. J Biol Chem. 2002;277(45):42419-22

20. Brown JR et al. Evolutionary relationships of aurora kinases: implications for model organism studies and the development of anti-cancer drugs. BMC Evol Biol. 2004;4:39.

21. Seeling JM et al. Differential selective pressures experienced by the Aurora kinase gene family. Int J Mol Sci. 2017;19(1):72.

22. Min $M$ et al. Ubiquitination site preferences in anaphase promoting complex/ cyclosome (APC/C) substrates. Open Biol. 2013:3(9):130097.

23. Setny P. Conserved internal hydration motifs in protein kinases. Proteins.
2020;88(12):1578-91.

24. Polverino F et al. The Aurora-A/TPX2 axis directs spindle orientation by regulating NuMa and microtubule dynamics. SSRN Electronic Journal. 2020;DOI:10.2139/ ssrn.3588889.

25. Zhao ZS et al. The GITassociated kinase PAK targets to the centrosome and regulates Aurora-A. Mol Cell. 2005;20(2):237-49.

26. Walter $A O$ et al. The mitotic serine/threonine kinase Aurora2/AIK is regulated by phosphorylation and degradation. Oncogene. 2000;19(42):4906-16.

27. Mori $\mathrm{D}$ et al. An essential role of the aPKC-Aurora A-NDEL1 pathway in neurite elongation by modulation of microtubule dynamics. Nat Cell Biol. 2009;11(9):1057-68.

28. Rannou $Y$ et al. Localization of Aurora A and Aurora B kinases during interphase: role of the $\mathrm{N}$-terminal domain. Cell Cycle. 2008;7(19):3012-20.

29. Tanaka M et al. Cell-cycledependent regulation of human Aurora A transcription is mediated by periodic repression of E4TF1. J Bio Chem. 2002;277(12):10719-26.

30. Rowan FC et al. Insights into Aurora-A kinase activation using unnatural amino acids incorporated by chemical modification. ACS Chem Biol. 2013;8(10):2184-91.

31. Chen $\mathrm{C}$ et al. AURKA promotes cancer metastasis by regulating epithelial-mesenchymal transition and cancer stem cell properties in hepatocellular carcinoma. Biochem Biophys Res Commun. 2017;486(2):514-20.

32. Liu $\mathrm{N}$ et al. Inhibition of Aurora A enhances radiosensitivity in selected lung cancer cell lines. Respir Res. 2019;20(1):230.

33. $\mathrm{Ma} Y$ et al. Aurora-A affects radiosenstivity in cervical squamous cell carcinoma and predicts poor prognosis. Oncotarget. 2017;8(19):31509-20.

34. Ma $Y$ et al. Mechanism of cervical squamous cell carcinoma radioresistance conferred by Aurora-A. Int J Clin Exp Pathol. 2017;10:2416-25.

35. Shen ZT et al. Aurora-A confers radioresistance in human hepatocellular carcinoma by activating NF-kB signaling pathway. BMC Cancer. 2019;19(1):1075.

36. Sun $\mathrm{H}$ et al. Aurora-A controls cancer cell radio- and chemoresistance via ATM/Chk2-mediated DNA repair networks. Biochim Biophys Acta. 2014;1843(5):934-44

37. Oh ET et al. Aurora-A contributes to radioresistance by increasing NF-kB DNA binding. Radiat Res. 2010;174(3):265-73.
38. Liu JB et al. Aurora-A/NF$\mathrm{KB}$ signaling is associated with radio-resistance in human lung adenocarcinoma. Anticancer Res. 2019;39(11):5991-8.

39. Wang $\mathrm{H}$ et al. Hypoxic radioresistance: can $\mathrm{ROS}$ be the key to overcome it? Cancers (Basel). 2019;11(1):112.

40. Cui SY et al. The role of Aurora A in hypoxia-inducible factor la-promoting malignant phenotypes of hepatocelluar carcinoma. Cell Cycle. 2013;12(17):2849-66.

41. Romain CV et al. Targeting Aurora kinase A inhibits hypoxiamediated neuroblastoma cell tumorigenesis. Anticancer Res. 2014;34(5):2269-74.

42. Asteriti IA et al. The Aurora-A inhibitor MLN8237 affects multiple mitotic processes and induces dose-dependent mitotic abnormalities and aneuploidy. Oncotarget. 2014;5(15):6229-42.

43. Liu $\mathrm{N}$ et al. Inhibition of Aurora A enhances radiosensitivity in selected lung cancer cell lines. Respir Res. 2019;20(1):230

44. Woo JK et al. Daurinol enhances the efficacy of radiotherapy in lung cancer via suppression of Aurora kinase A/B expression. Oncotarget. 2014;5(15):6229-42

45. Shiomitsu $\mathrm{K}$ et al.

The radiosensitizing effect of the Aurora kinase inhibitors, ENMD-2076, on canine mast cell tumours in vitro. Vet Comp Oncol. 2016;14(1):13-27.

46. Sun H, Yang G. Mechanistic study of pancreatic cancer cell radioresistance conferred by Aurora-A. China Oncology. 2013;(12):974-9.

47. Guan $Z$ et al. Aurora-A, a negative prognostic marker, increases migration and decreases radiosensitivity in cancer cells. Cancer Res. 2007;67(21):10436-44.

48. Yu CC et al. Targeting the PI3K/ AKT/mTOR signaling pathway as an effectively radiosensitizing strategy for treating human oral squamous cell carcinoma in vitro and in vivo. Oncotarget. 2017;8(40):68641-53.

49. Saigusa $\mathrm{S}$ et al. Correlation of CD133, OCT 4 , and SOX2 in rectal cancer and their association with distant recurrence after chemoradiotherapy. Ann Surg Oncol. 2009;16(12):3488-98.

50. Wang $\mathrm{J}$ et al. The Aurora-A-Twist1 axis promotes highly aggressive phenotypes in pancreatic carcinoma J Cell Sci. 2017;130(6):1078-93.

51. Puisieux $A$ et al. A twist for survival and cancer progression. $\mathrm{Br} \mathrm{J}$ Cancer. 2006:94(1):13-7.

52. Dar AA et al. The Aurora kinase A regulates GSK-3 $\beta$ in gastric cancer cells. Oncogene. 2009;28(6):866-75.

53. Le Grand $M$ et al. Interplay 
between $\mathrm{MycN}$ and c-Myc regulates radioresistance and cancer stem cell phenotype in neuroblastoma upon glutamine deprivation. Theranostics. 2020;10(14):6411-29.

54. Long $Y$ et al. How do IncRNAs regulate transcription? Sci Adv. 2017;3(9):eaao2110.

55. Fan $L$ et al. Long noncoding RNA MALAT1 contributes to sorafenib resistance by targeting $\mathrm{miR}$ 140-5p/Aurora-A signaling in hepatocellular carcinoma. Mol Cancer Ther. 2020;19(5):1197-209.

56. Li T et al. LncRNA TUG1 promotes cells proliferation and inhibits cells apoptosis through regulating AURKA in epithelial ovarian cancer cells. Medicine (Baltimore). 2018;97(36):12131.

57. Jiang $\mathrm{H}$ et al. Down-regulation of LnCRNA TUG1 enhances radiosensitivity in bladder cancer via suppressing HMGB1 expression Radiat Oncol. 2017;12(1):65.

58. Shen ZT et al. Aurora-A confers radioresistance in human hepatocellular carcinoma by activating NF-KB signaling pathway. BMC Cancer. 2019;19(1):1075.

59. Otto $T$ et al. Cell cycletargeting microRNAs promote differentiation by enforcing cellcycle exit. Proc Natl Acad Sci USA. 2017;114(40):10660-5.

60. Chan JY et al. Multiomic analysis and immunoprofiling reveal distinct subtypes of human angiosarcoma. $J$ Clin Invest. 2020;130(11):5833-46.

61. Carpinelli P et al. PHA-739358, a potent inhibitor of aurora kinases with a selective target inhibition profile relevant to cancer. Mol Cancer Ther. 2007;6(12 Pt 1):3158-68.

62. Tao $\mathrm{Y}$ et al. Enhancement of radiation response by inhibition of Aurora-A kinase using siRNA or a selective Aurora kinase inhibitor PHA680632 in p53-deficient cancer cells. $\mathrm{Br} \mathrm{J}$ Cancer. 2007:97(12):1664-72.

63. Wang $\mathrm{S}$ et al. Discovery of $\mathrm{N}$-phenyl4-(thiazol-5-yl)pyrimidin-2-amine aurora kinase inhibitors. J Med Chem. 2010;53(11):4367-78

64. Arbitrario JP et al. SNS-314, a pan-Aurora kinase inhibitor, shows potent anti-tumor activity and dosing flexibility in vivo. Cancer Chemother Pharmacol. 2010;65(4):707-17.

65. McLaughlin J et al. Preclinical characterization of Aurora kinase inhibitor R763/AS703569 identified through an image-based phenotypic screen. J Cancer Res Clin Oncol. 2010;136(1):99-113.

66. Cota CD et al. Cyclin-dependent kinase 1 and Aurora kinase choreograph mitotic storage and redistribution of a growth factor receptor. PLoS Biol. 2021:19(1):e3001029.
67. Howard S et al. Fragment-based discovery of the pyrazol-4-yl urea (AT9283), a multitargeted kinase inhibitor with potent aurora kinase activity. J Med Chem. 200;52(2):379-88.

68. Jani P J et al. PF-03814735, an orally bioavailable small molecule aurora kinase inhibitor for cancer therapy. Mol Cancer Ther. 2010;9(4):883-94.

69. Görgün $\mathrm{G}$ et al. A novel Aurora-A kinase inhibitor MLN8237 induces cytotoxicity and cell-cycle arrest in multiple myeloma. Blood. 2010;115(25):5202-13.

70. Wang $X$ et al. Preclinical activity of a novel multiple tyrosine kinase and aurora kinase inhibitor, ENMD2076, against multiple myeloma. $\mathrm{Br}$ J Haematol. 2010;150(3):313-25.

71. Manfredi MG et al. Antitumor activity of MLN8054, an orally active small-molecule inhibitor of Aurora A kinase. Proc Natl Acad Sci USA. 2007:104(10):4106-11.

72. Moretti L et al. MLN8054, a small molecule inhibitor of aurora kinase a, sensitizes androgen-resistant prostate cancer to radiation. Int J Radiat Oncol Biol Phys. 2011;80(4):1189-97. 